\title{
Article \\ Association between Physical Frailty Subdomains and Oral Frailty in Community-Dwelling Older Adults
}

\author{
Ryo Komatsu ${ }^{1}\left(\mathbb{D}\right.$, Koutatsu Nagai $^{1, *(\mathbb{D})}$, Yoko Hasegawa ${ }^{2,3}{ }^{(\mathbb{D}}$, Kazuki Okuda $^{1}$, Yuto Okinaka ${ }^{1}$, Yosuke Wada ${ }^{4}$, \\ Shotaro Tsuji ${ }^{5}$, Kayoko Tamaki ${ }^{6}$, Hiroshi Kusunoki ${ }^{6}$, Hiromitsu Kishimoto ${ }^{2}$ and Ken Shinmura ${ }^{6}$
}

check for updates

Citation: Komatsu, R.; Nagai, K.; Hasegawa, Y.; Okuda, K.; Okinaka, Y.; Wada, Y.; Tsuji, S.; Tamaki, K.; Kusunoki, H.; Kishimoto, H.; et al. Association between Physical Frailty Subdomains and Oral Frailty in Community-Dwelling Older Adults. Int. J. Environ. Res. Public Health 2021, 18, 2931. https://doi.org/10.3390/ ijerph18062931

Academic Editor: Giorgio Rappelli

Received: 11 February 2021

Accepted: 10 March 2021

Published: 12 March 2021

Publisher's Note: MDPI stays neutral with regard to jurisdictional claims in published maps and institutional affiliations.

Copyright: (c) 2021 by the authors. Licensee MDPI, Basel, Switzerland. This article is an open access article distributed under the terms and conditions of the Creative Commons Attribution (CC BY) license (https:/ / creativecommons.org/licenses/by/ $4.0 /)$
1 Department of Physical Therapy, School of Rehabilitation, Hyogo University of Health Sciences, 1-3-6 Minatojima, Chuo-ku, Kobe, Hyogo 650-8530, Japan; pr17024@std.huhs.ac.jp (R.K.); pr17012@std.huhs.ac.jp (K.O.); pr17011@std.huhs.ac.jp (Y.O.)

2 Department of Dentistry and Oral Surgery, Hyogo College of Medicine, 1-1 Mukogawa-cho, Nishinomiya, Hyogo 663-8501, Japan; cem17150@dent.niigata-u.ac.jp (Y.H.); kisihiro@hyo-med.ac.jp (H.K.)

3 Division of Comprehensive Prosthodontics, Faculty of Dentistry, Graduate School of Medical and Dental Sciences, Niigata University, Niigata 951-8514, Japan

4 Department of Rehabilitation, Hyogo College of Medicine Sasayama Medical Center, Sasayama, Hyogo 669-2321, Japan; yu-wada@hyo-med.ac.jp

5 Department of Orthopaedic Surgery, Hyogo College of Medicine, Nishinomiya, Hyogo 663-8501, Japan; tj13041305sho@gmail.com

6 Department of General Medicine, Hyogo College of Medicine, 1-1 Mukogawa, Nishinomiya, Hyogo 663-8501, Japan; ka-tamaki@hyo-med.ac.jp (K.T.); kusunoki1019@yahoo.co.jp (H.K.); ke-shimmura@hyo-med.ac.jp (K.S.)

* Correspondence: nagai-k@huhs.ac.jp; Tel.: +81-78-304-3047; Fax: +81-78-304-2747

Abstract: This cross-sectional study aimed to demonstrate the association between physical frailty subdomains and oral frailty. This study involved community-dwelling older adults (aged $\geq 65$ years). Physical frailty was assessed with the Japanese version of the Cardiovascular Health Study criteria. Oral frailty was defined as limitations in at least three of six domains. Logistic regression analysis was used to analyze the association between physical frailty risk and oral frailty. In addition, we examined the association between physical frailty subdomains (gait speed, grip strength, exhaustion, low physical activity, and weight loss) and oral frailty. A total of 380 participants were recruited for this study. Overall, $18 \%$ and $14 \%$ of the participants were at risk of physical frailty and had oral frailty, respectively. Physical frailty risk (odds ratio $(\mathrm{OR})=2.40,95 \%$ confidence interval $(\mathrm{CI}): 1.22-4.75$, $p=0.012$ ) was associated with oral frailty in multivariate analysis. In secondary analysis, among physical frailty subdomains, gait speed (OR $=0.85,95 \% \mathrm{CI}$ : $0.73-0.97, p=0.019)$ was associated with oral frailty. The present findings suggest that physical frailty is closely related to oral frailty. Among physical frailty subdomains, decreased gait speed in particular is an important indicator related to the development of oral frailty.

Keywords: physical frailty; oral frailty; physical performance; older adults

\section{Introduction}

According to the Fried phenotype, physical frailty is defined as physical and functional vulnerability [1]. Physical frailty includes low gait speed, weakness, exhaustion, low physical activity, and weight loss; as these domains are interrelated, a decline in one tends to drive a decline in another [2]. Physical frailty increases the risk of long-term care needs, falls, premature mortality, hospitalization, and disability $[1,3,4]$. Consequently, maintaining and improving overall health is important to preventing frailty and the associated consequences.

In addition, oral frailty is an important indicator of overall health. Oral frailty is defined as poor oral status that increases the risk of physical frailty, sarcopenia, long-term care needs, and premature mortality [5]. Moreover, oral frailty has been associated with social frailty and malnutrition, both of which increase the risk of physical frailty [6-11]. 
This evidence suggests that oral frailty is a significant contributor to health and thus a target for prevention.

A previous study has demonstrated that oral frailty may affect physical frailty [5]. Meanwhile, a previous study has shown that skeletal muscle mass index and grip strength among women might predict dysphagia [12]. In this way, physical and oral frailty may be associated with each other. This finding suggests that an intervention to improve physical function may ameliorate oral function.

In the present study, we aimed to examine the relationship between physical frailty and oral frailty. Although previous studies have shown a relationship between several domains of physical frailty (grip strength, gait speed, and physical activity) and oral health status (tongue pressure and chewing ability) $[13,14]$, the impact of each physical frailty subdomain on overall oral health remains unclear. The physical frailty subdomains are independent concepts; therefore, clarifying the association between physical frailty subdomains and oral frailty will facilitate the development of interventions corresponding to each domain. Herein, we aimed to clarify the relationship between physical frailty subdomains and oral frailty to provide evidence that may be used to interject the interrelationship between oral and physical health decline.

\section{Materials and Methods}

This cross-sectional study involved community-dwelling older adults living in the Sasayama-Tamba Area, which is a rural area in the Hyogo prefecture, Japan. The population of Tamba-Sasayama city was 41,490 as of 2015; the average age tends to be higher in this prefecture than in Japan overall (31.4\% of the population were aged $\leq 65$ years as of September 2015). Tamba-Sasayama City is located in the mountainous region of the Hyogo prefecture, where the leading sectors remain farming and agriculture. Data used in the present study were collected between April 2016 and December 2019. To recruit participants, we used newspaper inserts and poster advertisements at the Hyogo College of Medicine Sasayama Medical Center. We included individuals aged $\geq 65$ years. Exclusion criteria were individuals who (1) had missing data, and (2) had decreased cognitive function (Mini-Mental State Examination (MMSE) score <20) [15]. The data used in this study were anonymized. This study was approved by the institutional review board of the Hyogo College of Medicine (No. Rinhi 0342). All participants took part in this study voluntarily and provided written informed consent to be included. This study was conducted in compliance with the Helsinki Declaration.

\subsection{Participant Characteristics}

We assessed the following characteristics: age, height, body mass index (BMI), number of prescribed medications, comorbidities, cognitive function, education, and social activity. BMI was calculated by dividing body weight $(\mathrm{kg})$ by the height $(\mathrm{m})$ squared. Cognitive function was assessed using the MMSE. Social activity was evaluated by a response of "yes" or "no" using the following question from the Japan Science and Technology Agency Index of Competence [16]: "Do you participate in any community activities or volunteer activities?"

\subsection{Physical Frailty}

We considered physical frailty risk by limitations in at least two of the following conditions, the reference to the Japanese version of the Cardiovascular Health Study (J-CHS): [17] low gait speed, weakness, exhaustion, low physical activity, and weight loss. To assess gait speed, we asked participants to walk a $12 \mathrm{~m}$ walkway at their normal speed and measured the time for a $10 \mathrm{~m}$ walk. We established slow gait speed according to a cutoff point of $<1.0 \mathrm{~m} / \mathrm{s}$ [18]. Weakness was assessed using maximum grip strength, which was measured in kilograms using a Smedley-type handheld dynamometer (GRIP-A; Takei Ltd., Niigata, Japan). Weakness was established according to a sex-specific cutoff ( $<26 \mathrm{~kg}$ for men and $<18 \mathrm{~kg}$ for women) [19]. We considered exhaustion if participants 
responded "yes" to the following question from the Kihon Checklist (KCL) developed by the Japanese Ministry of Health, Labor, and Welfare (Supplementary Table S1) [20]: "In the last 2 weeks, have you felt tired without a reason?" Low physical activity was considered if participants responded "no" for the given question: [18] "Do you engage in low levels of physical exercise aimed at health?" Weight loss was assessed by a response of "yes" to the question, "Have you lost $2 \mathrm{~kg}$ or more in the past 6 months?" [20].

\subsection{Oral Frailty and Related Assessments}

We defined oral frailty as functional limitations in at least three of the following six domains [5]: few remaining teeth, reduced masticatory performance, reduced articulatory oral motor skills (examined using the ability to pronounce the "ta" sound), lower maximum tongue pressure, subjective difficulty eating hard foods, and subjective difficulty swallowing. The number of corresponding items was used as the oral frailty score. Participants whose oral frailty score was two or fewer were considered to be without oral frailty.

The number of remaining teeth was assessed by dentists. The cutoff value for few remaining teeth was of $<20$ [5]. We used a previously described scoring method to assess low masticatory performance [21]. Participants were instructed to chew a piece of test gummy jelly (UHA Mikakuto Co., Ltd., Osaka, Japan) freely 30 times; subsequently, the pieces of gummy jelly were objectively evaluated by a visual scoring method (on a scale from $0-9$ points). The cutoff value for low masticatory performance was a score of $\leq 2$ points [22]. Articulatory oral motor skills (pronunciation of the "ta" sound) were assessed using oral diadochokinesis ("ta"). Participants were asked to pronounce each syllable consecutively as fast as possible for $5 \mathrm{~s}$. We measured articulation counts per second using a digital counter (KENKOU-KUN ${ }^{\circledR}$; Takei Scientific Instruments Co., Ltd., Niigata, Japan). We established sex-specific cutoff values for oral diadochokinesis ("ta"), which were equivalent to $<5.2$ times/s and $<5.4$ times/s for men and women, respectively [5].

Lower maximum tongue pressure was measured at the midline of the dorsal surface of the tongue using a tongue pressure measurement device (JMS tongue pressure meter, JMS, Hiroshima, Japan). We established a sex-specific cutoff value for the lower maximum tongue pressure $(<27.4 \mathrm{kPa}$ and $26.5 \mathrm{kPa}$ for men and women, respectively) [5]. We considered subjective difficulty in eating hard foods to be present in participants that responded "yes" to the following question from KCL (Supplementary Table S1) [20]: "Compared to 6 months ago, do you find it more difficult to eat hard foods?" Subjective difficulty in swallowing was considered present in participants that responded "yes" to the following question: "Do you choke on tea or soup?" [20].

In addition to the oral frailty criteria, occlusal support was assessed using the Eichner classification, which was divided into 10 levels (A1-C3) according to the number of occlusal teeth pairs (the A1 level contained most pairs) [23].

\subsection{Statistics}

Continuous variables were assessed for the normality of distribution using the ShapiroWilk test. We used the Mann-Whitney U test to compare age, height, BMI, number of medications, the MMSE scores, education level, gait speed, and grip strength between oral robust and frailty. Differences in sex, comorbidities, history of falls, social activity levels, physical frailty risk, and physical frailty subdomains between the groups were assessed using the $\chi^{2}$ test.

We conducted two types of analysis using oral frailty as the dependent variable. Logistic regression analysis was used to analyze the association between physical frailty risk and oral frailty. Physical frailty risk as the independent variable was included in the crude model. Further, we included age, gender, the MMSE score, BMI, number of medications, and number of comorbidities as covariates, provided they were significantly associated with oral function in univariate models; we used the forced entry method in the adjusted model. In addition, to examine the relationship between components of physical frailty and oral frailty, we conducted an analysis including five physical frailty subdomains 
as independent variables, following J-CHS: gait speed, grip strength, exhaustion, low physical activity, and weight loss. We aimed to explore the factors of the physical frailty subdomain associated with oral frailty; hence, we included these as independent variables using the stepwise method in the crude model. Gait speed and grip strength were used as continuous values. The covariates (those defined in the first analysis) were included using the forced entry method in the adjusted model.

All analyses were performed with IBM SPSS version 24 (IBM Japan Ltd., Tokyo, Japan). $p$-Values of $<0.05$ were considered indicative of statistically significant findings.

\section{Results}

A total of 382 community-dwelling older adults who met the eligibility criteria were included in this study. Two participants lacked data required for multivariate analysis and were thus excluded. The final sample included 380 participants (116 men; mean age $72.8 \pm 5.6$ years). Sixty-nine (18\%) and $54(14 \%)$ participants had physical frailty risk and oral frailty, respectively (Table 1). The prevalence of low gait speed, weakness, exhaustion, low physical activity, and weight loss was $18(4.7 \%), 24(6.3 \%), 76(20 \%), 134(35 \%)$, and $54(14 \%)$, respectively. Nineteen $(35 \%)$ older adults with oral frailty showed physical frailty risk. The prevalence of oral frailty in each physical frailty subdomain was $6(11 \%)(p$-value for the difference between the oral robust and frailty group $=0.017), 5(9.3 \%)(p=0.337)$, $18(33 \%)(p=0.008), 26(48 \%)(p=0.032)$, and $12(22 \%)(p=0.069)$ for low gait speed, weakness, exhaustion, low physical activity, and weight loss, respectively. There were significant between-group differences in age $(p<0.001)$, cardiovascular disease prevalence $(p=0.002)$, education level $(p=0.038)$, social activity level $(p=0.002)$, and gait speed $(p=0.001)$ between participants with oral robust and with oral frailty.

Table 1. Characteristics in participants with oral frailty.

\begin{tabular}{|c|c|c|c|c|}
\hline Variables & $\begin{array}{l}\text { Overall } \\
(n=380)\end{array}$ & $\begin{array}{l}\text { Oral Robust } \\
\quad(n=326)\end{array}$ & $\begin{array}{l}\text { Oral Frailty } \\
\quad(n=54)\end{array}$ & $p$-Value \\
\hline Age, years, mean (SD) & $72.8(5.5)$ & $72.2(5.3)$ & $76.4(6.0)$ & $<0.001$ \\
\hline Female, $n(\%)$ & $264(69)$ & $224(69)$ & $40(74)$ & 0.428 \\
\hline Height, cm, mean (SD) & $155.5(8.3)$ & $155.8(8.2)$ & $153.6(8.5)$ & 0.069 \\
\hline Body mass index, $\mathrm{kg} / \mathrm{m}^{2}$, mean (SD) & $22.9(2.9)$ & $22.9(2.9)$ & $23.0(3.0)$ & 0.854 \\
\hline Medication, $n$, median (IQR) & $1.5(0-3)$ & $1.0(0-3)$ & $2.0(0-4)$ & 0.068 \\
\hline \multicolumn{5}{|l|}{ Comorbidities, $n(\%)$} \\
\hline Hypertension & $170(45)$ & $142(44)$ & $28(52)$ & 0.256 \\
\hline Diabetes & $44(12)$ & $38(11)$ & $6(11)$ & 0.919 \\
\hline Kidney disease & $18(4.7)$ & $16(4.9)$ & $2(3.7)$ & 0.700 \\
\hline Cardiovascular disease & $26(6.8)$ & $17(5.2)$ & $9(17)$ & 0.002 \\
\hline Osteoporosis & $50(13)$ & $42(13)$ & $8(15)$ & 0.697 \\
\hline MMSE, median (IQR) & $29(27-30)$ & $29(27-30)$ & $28(27-30)$ & 0.436 \\
\hline Education, years, median (IQR) & $12(12-14)$ & $12(12-14)$ & $12(9-14)$ & 0.038 \\
\hline Social activity, $n(\%)$ & $102(27)$ & $78(24)$ & $24(44)$ & 0.002 \\
\hline Physical frailty, $n(\%)$ & & & & $<0.001$ \\
\hline Robust & $311(82)$ & $278(85)$ & $33(65)$ & \\
\hline Frailty risk (>2 points) & $69(18)$ & $50(15)$ & $19(35)$ & \\
\hline \multicolumn{5}{|l|}{ Physical frailty subdomains, $n(\%)$} \\
\hline Low gait speed & $18(4.7)$ & $12(3.7)$ & $6(11)$ & 0.017 \\
\hline Weakness & $24(6.3)$ & $19(5.8)$ & $5(9.3)$ & 0.337 \\
\hline Exhaustion & $76(20)$ & $58(18)$ & $18(33)$ & 0.008 \\
\hline Low physical activity & $134(35)$ & $108(33)$ & $26(48)$ & 0.032 \\
\hline Weight loss & $54(14)$ & $42(13)$ & $12(22)$ & 0.069 \\
\hline Gait speed, m/s, mean (SD) & $1.4(0.2)$ & $1.5(0.2)$ & $1.3(0.2)$ & 0.001 \\
\hline Grip strength, kg, mean (SD) & $27.3(8.0)$ & $27.7(7.7)$ & $25.4(9.0)$ & 0.055 \\
\hline
\end{tabular}

SD, standard deviation; IQR, interquartile range; MMSE, Mini-Mental State Examination. $\chi^{2}$ test for proportions and nominal variables, Mann-Whitney U test for nonparametric variables. 
The prevalence of each oral frailty subdomain was as follows: 100 (26\%), 73 (19\%), $63(17 \%), 77(20 \%), 63(17 \%)$, and 84 (22\%) for few remaining teeth, low masticatory performance, low articulatory oral motor skill (pronunciation of the "ta" sound), lower maximum tongue pressure, subjective difficulty in eating hard foods, and subjective difficulty swallowing, respectively (Table 2).

Table 2. Distribution of participants per oral functions and statuses.

\begin{tabular}{|c|c|c|c|c|}
\hline Variables & $\begin{array}{c}\text { Overall } \\
(n=380)\end{array}$ & $\begin{array}{l}\text { Oral Robust } \\
(n=326)\end{array}$ & $\begin{array}{l}\text { Oral Frailty } \\
\quad(n=54)\end{array}$ & $p$-Value \\
\hline \multicolumn{5}{|l|}{ Oral frailty subdomains, $n(\%)$} \\
\hline Few remaining teeth & $100(26)$ & $57(18)$ & $43(80)$ & $<0.001$ \\
\hline Low masticatory performance & $73(19)$ & $36(11)$ & $37(69)$ & $<0.001$ \\
\hline Low articulatory oral motor skill & $63(17)$ & $39(12)$ & $24(44)$ & $<0.001$ \\
\hline $\begin{array}{l}\text { (pronunciation of "ta" sound) } \\
\text { Lower maximum tongue pressure }\end{array}$ & $77(20)$ & $53(16)$ & $24(44)$ & $<0.001$ \\
\hline Subjective difficulty in eating hard foods & $63(17)$ & $30(9.2)$ & $33(61)$ & $<0.001$ \\
\hline Subjective difficulty in swallowing & $84(22)$ & $55(17)$ & $29(54)$ & $<0.001$ \\
\hline Eichner classification, $n(\%)$ & & & & $<0.001$ \\
\hline A1 & $58(15)$ & $58(18)$ & $0(0)$ & \\
\hline A2 & $81(21)$ & $77(24)$ & $4(7)$ & \\
\hline A3 & $46(12)$ & $45(14)$ & $1(2)$ & \\
\hline B1 & $62(16)$ & $58(18)$ & $4(7)$ & \\
\hline B2 & $43(11)$ & $37(11)$ & $6(11)$ & \\
\hline B3 & $32(8)$ & $20(6)$ & $12(22)$ & \\
\hline $\mathrm{B} 4$ & $15(4)$ & $10(3)$ & $5(9)$ & \\
\hline C1 & $7(2)$ & $5(2)$ & $2(4)$ & \\
\hline $\mathrm{C} 2$ & $22(6)$ & $10(3)$ & $12(22)$ & \\
\hline $\mathrm{C} 3$ & $14(4)$ & $6(2)$ & $8(15)$ & \\
\hline
\end{tabular}

$\chi^{2}$ test for proportions and nominal variables, Mann-Whitney U test for ordinal variables.

The prevalence of each oral frailty subdomain among older adults with oral frailty was as follows: 43 (80\%), 37 (69\%), 24 (44\%), $24(44 \%), 33(61 \%)$, and $29(54 \%)$ for few remaining teeth, low masticatory performance, low articulatory oral motor skill (pronunciation of the "ta" sound), lower maximum tongue pressure, subjective difficulty in eating hard foods, and subjective difficulty swallowing, respectively. Eichner classifications were significantly different between the oral robust and frailty groups $(p<0.001)$.

Logistic regression analysis revealed that physical frailty risk (odds ratio $(\mathrm{OR})=3.00$, $95 \%$ confidence interval (CI): $1.59-5.65, p=0.001)$ was associated with oral frailty in model 1 (Table 3). In model 2 (adjusted model), physical frailty risk (OR: 2.40, 95\% CI: 1.22-4.75, $p=0.012)$ remained significantly associated with oral frailty.

Table 3. Relationship between physical and oral frailty.

\begin{tabular}{lcccccc}
\hline & \multicolumn{3}{c}{ Model 1 } & \multicolumn{3}{c}{ Model 2 } \\
\cline { 2 - 7 } & OR & $\mathbf{9 5 \% ~ C I ~}$ & $p$-Value & OR & $\mathbf{9 5 \% ~ C I ~}$ & $p$-Value \\
\hline $\begin{array}{l}\text { Overall }(n=380) \\
\begin{array}{l}\text { Physical frailty risk } \\
\text { Physical frailty subdomains }\end{array}\end{array}$ & 3.00 & $1.59-5.65$ & 0.001 & 2.40 & $1.22-4.75$ & 0.012 \\
$\quad$ Exhaustion & & & & & & \\
$\quad$ Gait speed & 2.19 & $1.15-4.17$ & 0.017 & 1.93 & $0.96-3.87$ & 0.062 \\
& 0.80 & $0.70-0.92$ & 0.001 & 0.85 & $0.73-0.97$ & 0.019 \\
\hline
\end{tabular}

Model 1 was a crude model without any adjustments. Physical frailty subdomains were added as independent variables in stepwise analysis. Model 2 was adjusted for age, gender, cardiovascular disease, MMSE, body mass index (BMI), and number of medications prescribed, using the forced entry method. OR, odds ratio.

In the analysis of oral and physical frailty subdomains, gait speed $(\mathrm{OR}=0.80,95 \% \mathrm{CI}$ : $0.70-0.92, p=0.001)$ and exhaustion $(\mathrm{OR}=2.19,95 \% \mathrm{CI}: 1.15-4.17, p=0.017)$ were significantly associated with oral frailty in model 1 (Table 3). Significant relationships in model 1 were not detected in weakness, low physical activity, and weight loss with oral frailty. In model 2 (adjusted model), gait speed (OR $=0.85,95 \% \mathrm{CI}: 0.73-0.97, p=0.019$ ) remained significantly associated with oral frailty. Exhaustion was not significantly associated with oral frailty in model 2. 


\section{Discussion}

In this study, we investigated the association between physical and oral frailty. In addition, we attempted to identify physical frailty subdomains that were significantly associated with oral frailty. In the present study, physical frailty risk was associated with oral frailty. Moreover, gait speed, a physical frailty subdomain, was associated with oral frailty.

The prevalence of oral frailty was $14 \%$ in the present study, which was similar to that previously reported [5-7]. Among the subdomains of oral frailty, the item representing few remaining teeth was the most prevalent. Factors associated with tooth loss include periodontal disease, dental caries, prosthodontic treatment, age, low socioeconomic status, diabetes mellitus, mean initial bone loss, and smoking [24-26]. Among these factors, periodontal disease and dental caries are considered major risk factors for tooth loss [27]. Compared to other subdomains in oral frailty, having few remaining teeth was the most prevalent $(80 \%)$, which suggests that prioritizing tooth loss prevention may be a key to avert the development of oral frailty.

The present study has shown an association between physical frailty risk and oral frailty. A previous study reported higher prevalence of oral frailty among older adults with physical pre-frailty signs than among those without such signs [6]. A longitudinal study by Tanaka et al. has shown that oral frailty is a risk factor for the future development of physical frailty [5]. In addition to studies that investigated the overall relationship between oral and physical function using the frailty concept, several studies have demonstrated relationships between physical (grip strength, gait speed, and physical activity) and oral (tongue pressure and chewing ability) frailty components $[13,14]$. The present study findings are consistent with those of these previous studies, providing additional evidence for the relationship between oral and physical frailty.

Gait speed, a subdomain of physical frailty, was significantly associated with oral frailty. Oral frailty may cause physical frailty, as the former may affect nutritional status among older adults and lead to weight loss [8]. Xue et al. have suggested a frailty cycle model, whereby weight loss leads to weakness, exhaustion, and low gait speed [2]. In the present study, weight loss and grip strength between the oral robust and frailty groups were not significantly different even in the univariate analysis (weight loss: $p=0.069$, grip strength: $p=0.055$ ). Since lower gait speed was the only significant factor, it is difficult to determine the relationship between other subdomains (weight loss, weakness, fatigue, and muscle weakness) and oral frailty in this study. Further research is needed on the relationship between the frailty cycle and oral frailty. Additionally, the fact that only lower gait speed was statistically significant suggests that oral frailty may be a sensitive indicator of declined gait speed. Meanwhile, tooth loss may accelerate the decline of gait speed [28]. However, this pathway is likely complex and hierarchical and thus difficult to assess in a cross-sectional design. Nevertheless, the present result suggests the necessity to assess both oral function and gait performance in clinical practice.

These findings notwithstanding, the association between physical and oral frailty may have the reverse direction. Lower gait speed is a risk factor for social frailty, whereby limited mobility may reduce the likelihood of social interactions [29]. Social frailty, which may include reduced social activity or contact with other people, may reduce opportunities for verbal communications among older adults, which in turn may affect oral function as a result of the reduced use of the muscles surrounding the pharynx and mouth [30]. In the present study, a significant difference in social activity was observed between the oral robust and frailty groups in the univariate analysis, indicating a possibility that low gait speed may increase the risk of oral frailty. Longitudinal studies are required to elucidate the causal relationship between physical and oral frailty.

This study has three limitations. First, it was a cross-sectional study, which precluded any meaningful discussions of causality. Second, the present participants volunteered to be included in this study, which may have resulted in selection bias. Third, in this study the criterion for physical frailty was operationally defined as two or more items being 
applicable, referring to the criteria of previous studies. Therefore, it is different from the more commonly used definition of three or more items, which may limit the generalizability of results. Incidentally, in the present study, masticatory performance assessment was conducted using a different material from that of the previous study. Gummy jelly assesses chewing ability, as well as chewing gum. Meanwhile, an earlier study reported that chewing gum and gummy jelly could assess different types of ability, with bias toward mixing and shearing ability, respectively [31]. Although the properties of the two materials are strictly different, we assumed that the differences did not significantly influence the results from the perspective of assessing the same ability in a broad sense. These limitations notwithstanding, this is the first study to examine the relationship between oral frailty and physical frailty subdomains. Knowing these relationships is important to prevent and treat frailty in clinical settings.

\section{Conclusions}

In this study, physical frailty risk was significantly associated with oral frailty. In addition, lower gait speed (a physical frailty subdomain) was associated with increased prevalence of oral frailty. Further studies are required to clarify the causal relationship between physical and oral frailty to develop suitable prevention and treatment interventions.

Supplementary Materials: The following are available online at https:/ / www.mdpi.com/1660-4 601/18/6/2931/s1, Table S1: Kihon Checklist English version created by Japan Geriatrics Society Working Group on Frailty.

Author Contributions: Conceptualization, R.K., K.N., K.O., Y.O., and Y.H.; methodology, R.K., K.N., K.O., Y.O., and Y.H.; formal analysis, R.K., K.O., and Y.O.; investigation, R.K., K.N., K.O., Y.O., Y.H., and K.S.; data curation, K.N., Y.H., Y.W., S.T., K.T., H.K. (Hiroshi Kusunoki), H.K. (Hiromitsu Kishimoto), and K.S.; writing—original draft preparation, R.K., K.N., K.O., and Y.O.; writing—review and editing, Y.H., Y.W., S.T., K.T., H.K. (Hiroshi Kusunoki), H.K. (Hiromitsu Kishimoto), and K.S. All authors have read and agreed to the published version of the manuscript.

Funding: This study was supported by the Hyogo Dental Association, 8020 Foundation (Yoko Hasegawa), the Mitsui Sumitomo Insurance Welfare Award, a grant in the field of Senior Citizen Welfare (Ken Shinmura), and by the Japan Society for the Promotion of Science (JSPS) Grant-in-Aid for Scientific Research (KAKENHI) (grant no. 16KT0012 to Ken Shinmura, 19K19723 to Koutatsu Nagai, and 16K11640 to Yoko Hasegawa).

Institutional Review Board Statement: This study was conducted according to the guidelines of the Declaration of Helsinki, and was approved by the institutional review board of the Hyogo College of Medicine (No. Rinhi 0342).

Informed Consent Statement: Informed consent was obtained from all participants involved in the study.

Data Availability Statement: The data that support the findings of this study are available from the corresponding author, upon reasonable request.

Acknowledgments: We acknowledge Mayu Harayama and Natsumi Okawa for their contribution to data collection.

Conflicts of Interest: The authors declare no conflict of interest.

\section{References}

1. Fried, L.P.; Tangen, C.M.; Walston, J.; Newman, A.B.; Hirsch, C.; Gottdiener, J.; Seeman, T.; Tracy, R.; Kop, W.J.; Burke, G.; et al. Frailty in Older Adults: Evidence for a Phenotype. J. Gerontol. A Biol. Sci. Med. Sci. 2001, 56, M146-M156. [CrossRef]

2. Xue, Q.-L.; Bandeen-Roche, K.; Varadhan, R.; Zhou, J.; Fried, L.P. Initial Manifestations of Frailty Criteria and the Development of Frailty Phenotype in the Women's Health and Aging Study II. J. Gerontol. Ser. A 2008, 63, 984-990. [CrossRef]

3. Chen, S.; Honda, T.; Narazaki, K.; Chen, T.; Kishimoto, H.; Kumagai, S. Physical Frailty and Risk of Needing Long-Term Care in Community-Dwelling Older Adults: A 6-Year Prospective Study in Japan. J. Nutr. Health Aging 2019, 23, 856-861. [CrossRef]

4. Kojima, G. Frailty as a Predictor of Future Falls among Community-Dwelling Older People: A Systematic Review and MetaAnalysis. J. Am. Med. Dir. Assoc. 2015, 16, 1027-1033. [CrossRef] [PubMed] 
5. Tanaka, T.; Takahashi, K.; Hirano, H.; Kikutani, T.; Watanabe, Y.; Ohara, Y.; Furuya, H.; Tetsuo, T.; Akishita, M.; Iijima, K. Oral Frailty as a Risk Factor for Physical Frailty and Mortality in Community-Dwelling Elderly. J. Gerontol. A Biol. Sci. Med. Sci. 2018, 73, 1661-1667. [CrossRef] [PubMed]

6. Hironaka, S.; Kugimiya, Y.; Watanabe, Y.; Motokawa, K.; Hirano, H.; Kawai, H.; Kera, T.; Kojima, M.; Fujiwara, Y.; Ihara, K.; et al. Association between Oral, Social, and Physical Frailty in Community-Dwelling Older Adults. Arch. Gerontol. Geriatr. 2020, 89, 104105. [CrossRef]

7. Iwasaki, M.; Motokawa, K.; Watanabe, Y.; Shirobe, M.; Inagaki, H.; Edahiro, A.; Ohara, Y.; Hirano, H.; Shinkai, S.; Awata, S. Association between Oral Frailty and Nutritional Status Among Community-Dwelling Older Adults: The Takashimadaira Study. J. Nutr. Health Aging 2020, 24, 1003-1010. [CrossRef] [PubMed]

8. Iwasaki, M.; Motokawa, K.; Watanabe, Y.; Shirobe, M.; Inagaki, H.; Edahiro, A.; Ohara, Y.; Hirano, H.; Shinkai, S.; Awata, S. A Two-Year Longitudinal Study of the Association between Oral Frailty and Deteriorating Nutritional Status among CommunityDwelling Older Adults. Int. J. Environ. Res. Public Health 2021, 18, 213. [CrossRef]

9. Makizako, H.; Shimada, H.; Doi, T.; Tsutsumimoto, K.; Hotta, R.; Nakakubo, S.; Makino, K.; Lee, S. Social Frailty Leads to the Development of Physical Frailty among Physically Non-Frail Adults: A Four-Year Follow-Up Longitudinal Cohort Study. Int. J. Environ. Res. Public Health 2018, 15, 490. [CrossRef] [PubMed]

10. Verlaan, S.; Ligthart-Melis, G.C.; Wijers, S.L.J.; Cederholm, T.; Maier, A.B.; de van der Schueren, M.A.E. High Prevalence of Physical Frailty Among Community-Dwelling Malnourished Older Adults-A Systematic Review and Meta-Analysis. J. Am. Med. Dir. Assoc. 2017, 18, 374-382. [CrossRef] [PubMed]

11. Jung, H.; Kim, M.; Lee, Y.; Won, C.W. Prevalence of Physical Frailty and Its Multidimensional Risk Factors in Korean CommunityDwelling Older Adults: Findings from Korean Frailty and Aging Cohort Study. Int. J. Environ. Res. Public Health 2020, $17,7883$. [CrossRef]

12. Maeda, K.; Takaki, M.; Akagi, J. Decreased Skeletal Muscle Mass and Risk Factors of Sarcopenic Dysphagia: A Prospective Observational Cohort Study. J. Gerontol. Ser. A 2017, 72, 1290-1294. [CrossRef]

13. Wakasugi, Y.; Tohara, H.; Machida, N.; Nakane, A.; Minakuchi, S. Can Grip Strength and/or Walking Speed Be Simple Indicators of the Deterioration in Tongue Pressure and Jaw Opening Force in Older Individuals? Gerodontology 2017, 34, 455-459. [CrossRef] [PubMed]

14. Kamdem, B.; Seematter-Bagnoud, L.; Botrugno, F.; Santos-Eggimann, B. Relationship between Oral Health and Fried's Frailty Criteria in Community-Dwelling Older Persons. BMC Geriatr. 2017, 17, 174. [CrossRef]

15. Perneczky, R.; Wagenpfeil, S.; Komossa, K.; Grimmer, T.; Diehl, J.; Kurz, A. Mapping Scores Onto Stages: Mini-Mental State Examination and Clinical Dementia Rating. Am. J. Geriatr. Psychiatry 2006, 14, 139-144. [CrossRef] [PubMed]

16. Iwasa, H.; Masui, Y.; Inagaki, H.; Yoshida, Y.; Shimada, H.; Otsuka, R.; Kikuchi, K.; Nonaka, K.; Yoshida, H.; Yoshida, H.; et al. Assessing Competence at a Higher Level among Older Adults: Development of the Japan Science and Technology Agency Index of Competence (JST-IC). Aging Clin. Exp. Res. 2018, 30, 383-393. [CrossRef]

17. Yamada, M.; Nishiguchi, S.; Fukutani, N.; Aoyama, T.; Arai, H. Mail-Based Intervention for Sarcopenia Prevention Increased Anabolic Hormone and Skeletal Muscle Mass in Community-Dwelling Japanese Older Adults: The INE (Intervention by Nutrition and Exercise) Study. J. Am. Med. Dir. Assoc. 2015, 16, 654-660. [CrossRef]

18. Shimada, H.; Makizako, H.; Doi, T.; Yoshida, D.; Tsutsumimoto, K.; Anan, Y.; Uemura, K.; Ito, T.; Lee, S.; Park, H.; et al. Combined Prevalence of Frailty and Mild Cognitive Impairment in a Population of Elderly Japanese People. J. Am. Med. Dir. Assoc. 2013, 14, 518-524. [CrossRef]

19. Chen, L.-K.; Liu, L.-K.; Woo, J.; Assantachai, P.; Auyeung, T.-W.; Bahyah, K.S.; Chou, M.-Y.; Chen, L.-Y.; Hsu, P.-S.; Krairit, O.; et al. Sarcopenia in Asia: Consensus Report of the Asian Working Group for Sarcopenia. J. Am. Med. Dir. Assoc. 2014, 15, 95-101. [CrossRef] [PubMed]

20. Fukutomi, E.; Okumiya, K.; Wada, T.; Sakamoto, R.; Ishimoto, Y.; Kimura, Y.; Chen, W.-L.; Imai, H.; Kasahara, Y.; Fujisawa, M.; et al. Relationships between Each Category of 25-Item Frailty Risk Assessment (Kihon Checklist) and Newly Certified Older Adults under Long-Term Care Insurance: A 24-Month Follow-up Study in a Rural Community in Japan. Geriatr. Gerontol. Int. 2015, 15, 864-871. [CrossRef]

21. Nokubi, T.; Yoshimuta, Y.; Nokubi, F.; Yasui, S.; Kusunoki, C.; Ono, T.; Maeda, Y.; Yokota, K. Validity and Reliability of a Visual Scoring Method for Masticatory Ability Using Test Gummy Jelly. Gerodontology 2013, 30, 76-82. [CrossRef] [PubMed]

22. Hasegawa, Y.; Sakuramoto-Sadakane, A.; Nagai, K.; Tamaoka, J.; Oshitani, M.; Ono, T.; Sawada, T.; Shinmura, K.; Kishimoto, H. Does Oral Hypofunction Promote Social Withdrawal in the Older Adults? A Longitudinal Survey of Elderly Subjects in Rural Japan. Int. J. Environ. Res. Public Health 2020, 17, 8904. [CrossRef] [PubMed]

23. Eichner, K. [Renewed examination of the group classification of partially edentulous arches by Eichner and application advices for studies on morbidity statistics]. Stomatol. DDR 1990, 40, 321-325.

24. Stabholz, A.; Babayof, I.; Mersel, A.; Mann, J. The Reasons for Tooth Loss in Geriatric Patients Attending Two Surgical Clinics in Jerusalem, Israel. Gerodontology 1997, 14, 83-88. [CrossRef] [PubMed]

25. Müller, S.; Eickholz, P.; Reitmeir, P.; Eger, T. Long-Term Tooth Loss in Periodontally Compromised but Treated Patients According to the Type of Prosthodontic Treatment. A Retrospective Study. J. Oral Rehabil. 2013, 40, 358-367. [CrossRef]

26. Hanioka, T.; Ojima, M.; Tanaka, K.; Matsuo, K.; Sato, F.; Tanaka, H. Causal Assessment of Smoking and Tooth Loss: A Systematic Review of Observational Studies. BMC Public Health 2011, 11, 221. [CrossRef] 
27. Cahen, P.M.; Frank, R.M.; Turlot, J.C. A Survey of the Reasons for Dental Extractions in France. J. Dent. Res. 1985, 64, 1087-1093. [CrossRef]

28. Welmer, A.-K.; Rizzuto, D.; Parker, M.G.; Xu, W. Impact of Tooth Loss on Walking Speed Decline over Time in Older Adults: A Population-Based Cohort Study. Aging Clin. Exp. Res. 2017, 29, 793-800. [CrossRef]

29. Nagai, K.; Tamaki, K.; Kusunoki, H.; Wada, Y.; Tsuji, S.; Itoh, M.; Sano, K.; Amano, M.; Hayashitani, S.; Yokoyama, R.; et al. Physical Frailty Predicts the Development of Social Frailty: A Prospective Cohort Study. BMC Geriatr. 2020, 20, 403. [CrossRef]

30. Nagayoshi, M.; Higashi, M.; Takamura, N.; Tamai, M.; Koyamatsu, J.; Yamanashi, H.; Kadota, K.; Sato, S.; Kawashiri, S.; Koyama, Z.; et al. Social Networks, Leisure Activities and Maximum Tongue Pressure: Cross-Sectional Associations in the Nagasaki Islands Study. BMJ Open 2017, 7, e014878. [CrossRef]

31. Yamada, A.; Kanazawa, M.; Komagamine, Y.; Minakuchi, S. Association between Tongue and Lip Functions and Masticatory Performance in Young Dentate Adults. J. Oral Rehabil. 2015, 42, 833-839. [CrossRef] [PubMed] 